\title{
The design of lightweight gas turbine engine parts using topology optimization
}

\author{
Aleksandra Dubrovskaya ${ }^{1, *}$, Konstantin Dongauzer ${ }^{1}$, and Rustam Faskhutdinov ${ }^{1}$ \\ ${ }^{1}$ Perm national research polytechnic university, Computation Mathematics and Mechanics Department 29, Komsomolsky prospect, \\ Perm, 614990, Russian Federation
}

\begin{abstract}
This paper addresses a new approach to aircraft engine parts designing using the topological optimization algorithm for production by additive manufacturing. The full mathematical formulation which includes the problem statement within the elasticity theory and the SIMP optimization procedure is presented. The criterion particularities for evaluation of strength properties of the gas turbine engines parts according to the Aviation Regulations are specified. Optimization of the typical hinge bracket topology is performed, and the stress state of the part with the initial geometry and the optimized geometry is evaluated.
\end{abstract}

\section{Introduction}

Additive manufacturing is the newest production method which completely changes the approach to part designing. This method allows getting a complex structure with minimum post-process machining in one step literally. The structure complicity and shape intricacy of the part don't influence the production prime-cost, what matters is the weight of the part. Application of additive manufacturing allows a designer to eliminate limitations imposed by the strict rules of the technological capabilities in the production environment.

Even a slight weight reduction in an engine results in significant fuel savings and pollutant emissions reduction in the aerospace industry. That's why the industry's main objective is to design aircraft parts with lower weight while maintaining their prescribed functionality and life time. Rapid development of additive manufacturing allowed for recollection of a well-known, but up until now hardly applicable designing technology, that is topological optimization. Back then, manufacturing of optimized products was impractical and often impossible, as it is labour intensive and involves considerable investments in order to provide a complex geometry by conventional production methods. Fundamental nature of the topological optimization method performs the same idea, being the cornerstone of additive manufacturing, of getting the material exactly where it is needed. The methods of additive manufacturing and topological optimization united by the common concept are able to perform a leap-ahead in up-to-date domestic engine manufacturing.

The results of this work will be used in UECAviadvigatel JSC when building up high-tech manufacturing of complex-shaped parts for aircraft and industrial gas turbine engines based on additive manufacturing from Russian metal powders.

\section{Peculiarities in the evaluation of aircraft parts strength properties}

Aircraft parts can undergo an entire range of combinedloaded conditions during operation dependent on the engine behaviour. It is a critical and knowledge-intensive task to define and take into consideration all the load profiles, and to bring all the parts to conformity with section 25 "Airworthiness Requirements for Carrier Aircrafts" and section 33 "Airworthiness Requirements of Aircrafts Engines" of the Aviation Regulations.

The following static strength requirements are applied to aircraft parts:

- The parts shall withstand the maximum operation loads with no dangerous fatigue deformations showing.

- The parts shall withstand design loads (that is the operation loads multiplied by safety factors) with no failure during three seconds.

The additional safety factor for the additive manufactured parts is not clearly specified in the Aviation Regulations. In this case, it was decided to take the safety factors equal to 1,5 (the factor used for castings) to ensure strength properties.

The fatigue resistance in the part is evaluated based on the following rule:

- The parts life shall be not less than 40000 cycles (80000 hours).

\section{Theoretical basis of the topological optimization method}

Generation of the optimal geometry design model for the part must be started from the definition domain identification. For this purpose, the procedure of geometry extension within the dimensions meeting the conditions of the part mounting into the assembly, and simplification of the initial topology of the item to be

\footnotetext{
* Corresponding author: dubrovskaya-as@avid.ru
} 
designed are performed [1]. Static loading diagrams and loads variation rates are defined based on the operation force diagrams in different flight modes.

The topology optimization task is solved based on the calculated stress state of the part. As the aircraft parts must undergo no plastic strain during operation, it makes sense to chase the stress fields in 3D elastic formulation.

Let us consider a 3D elastic body with the volume $V_{0}$ and the boundary $B$ fixed in the space along the boundary $B_{0}$ and subjected to the external loads complex $\hat{F}$. To find the stress tensor $\hat{\sigma}$, let us to write down the general form of the quasi-static moment equilibrium equation for this case :

$$
-\nabla \cdot \hat{\sigma}=\nabla \hat{F} .
$$

The physical relations of the elasticity theory can be defined as:

$$
\hat{\sigma}={ }^{4} \hat{K} \cdot \hat{\varepsilon}^{e l},
$$

where ${ }^{4} K$ is a fourth-rank elastic constants tensor, and $\hat{\varepsilon}^{e l}$ is an elastic strain tensor.

Using the small-strain theory, let us write down the geometrical relations as:

$$
\hat{\varepsilon}=\frac{1}{2}\left[\nabla u+(\nabla u)^{T}\right],
$$

where $\bar{u}$ is a global displacement vector [2].

It is necessary to define the conditions of fixing $\pi_{0}$ at the boundary $B_{0}$, the boundary conditions for this task will then take on form of:

$$
\left.\bar{u}\right|_{\Gamma_{0}}=\bar{u}_{0} .
$$

For the special case of the rigid fixing, $\bar{u}_{0}=0$.

This task is solved by the finite elements method. Trigonal simplex elements with linear Lagrange approximation are used.

The SIMP (Solid Isotropic Materials with Penalization) method is used for topological optimization of a structure based on the specified stress fields. Its main idea is to get a tensor of the virtual specific binary densities in each element for effective material distribution. Initially, the specific density is prescribed as homogeneous and is equal to one in each element.

Let us assume that $\hat{x}$ is a tensor of the specific densities. The SIMP topological optimization method is based on prescription of the power laws between these densities and the elastic modulus. Such a dependency acts as a penalty function when related values of the power coefficient are more than one.

Let us assume that each element $i$ has a correspondent $E_{i 0}$ - which is a Young's modulus of the structure material in this element, and its own distribution density $x_{i}$, which defines the relative Young's modulus $E_{i}\left(x_{i}\right)$ for it. Thus, it is correct that:

$$
E_{i}\left(x_{i}\right)=E_{\min }+x_{i}^{p}\left(E_{i 0}-E_{\min }\right), x_{i} \in[0,1]
$$

where $p$ is a weight coefficient of penalty functions, used to ensure a black-and-white solution, and $E_{\min }$ is some small value of the Young's modulus related to the empty domain and introduced to eliminate the confluence of the elastic constants tensor, [3].

The solution of the task resolves into achievement of minimum interchanges in the finite-element mesh points. The target function is structure compliance $c$. It is an integral characteristic which is inverse to stiffness and is a function of a specific density. The solution to minimize the prescribed target function is sought:

$$
\min _{\hat{x}} c(\hat{x})=\bar{u}^{T} \cdot \hat{K} \cdot \bar{u}=\sum_{i=1}^{N} E_{i}\left(x_{i}\right) \bar{u}_{i}^{T} k_{i} \bar{u}_{i}
$$

where $\bar{u}_{i}$ and $k_{i}$ are a displacement vector and an element of the elastic constants tensor for the element $i$ respectively, $N$ is the number of the elements in the optimization domain.

However, the weight of the optimized product acts as a restriction to the target function, i.e. the variation range of the virtual density is controlled via the final part material volume $V(\hat{x})$ and the prescribed volume faction $f[4]$.

$$
\frac{V(\hat{x})}{V_{0}}=f
$$

Besides, the principle of virtual displacements acts as a restriction:

$$
{ }^{4} \hat{K} \cdot \bar{u}=\hat{F}
$$

The result of the SIMP method is black-and-white density distribution. That is, the tensor $\hat{x}$ takes on not only specific values, but also has a binary form. Though, the disadvantage of the method is the problem of Checkerboard patterns in the received solution. The received densities distribution doesn't form a closed domain, but contains unlinked segments.

In order to ensure the solution consistency and prevent occurrence of Checkerboard patterns, some restrictions shall be imposed on the optimized geometry. The most favourable approach is to apply a filter to sensitivities, i.e. to define the elements with the maximum influence to the target function. The sensitivity filter changes the sensitivity $\partial c / \partial x_{i}$ as follows:

$$
\frac{\hat{\partial c}}{\partial x_{i}}=\frac{1}{\max \left(\gamma, x_{i}\right) \sum_{j=1}^{N} H_{i j}} \sum_{j=1}^{N} H_{i j} x_{j} \frac{\partial c}{\partial x_{j}}
$$

where $N$ is a set of the elements $j$, for which the distance $\Delta(i, j)$ between the center of the current element $i$ is less than some critical radius of influence 
$r_{\min }, \gamma$ is an artificially implemented parameter equal to a small positive number which is necessary to exclude a possibility of zero-divide; and $H_{i j}$ is a weight coefficient, defined by:

$$
\hat{H}_{i j}=\max \left(0, r_{\min }-\Delta(i, j)\right)
$$

After the filter application, the original specific densities $x_{i}$, gained during optimization, are changed to:

$$
\widetilde{x}_{i}=\frac{1}{\sum_{j=1}^{N} H_{i j}} \sum_{j=1}^{N} H_{i j} x_{j}
$$

Therefore, the variables $x_{i}$ serve for preoptimization of the density in the domain of interest, while the filtered parameters $\widetilde{x}_{i}$ correlate to the actual physical density of the optimized structure [5]. This notation is used in order to highlight the fact that application of the densities filter results in $x_{i}$ losing its physical meaning. That's why the filtered binary specific densities $\widetilde{x}_{i}$ will be the solution of the optimization task.

\section{Application of the topological optimization method in aircraft parts designing}

In order to test the topological optimization technique and to evaluate its applicability in UEC-Aviadvigatel JSC, a typical hinge bracket used in the up-to-date domestic aircraft engine (Figure 1) was chosen. There are eight pieces of such brackets in the engine; each weighs 392 grams.

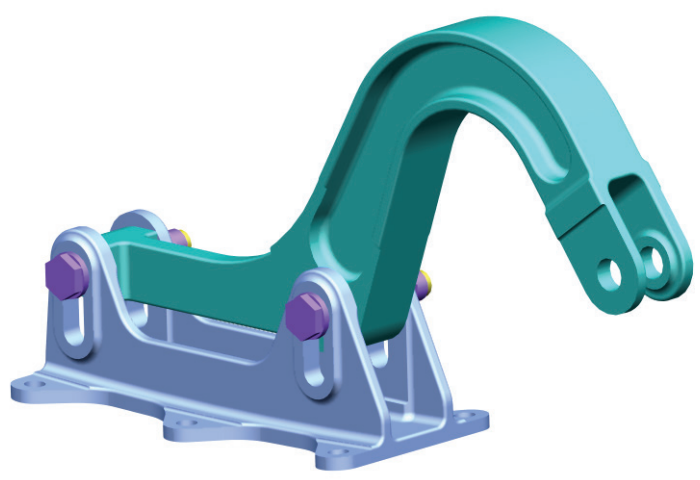

Fig. 1. An engineering model of the part chosen for designing with topological optimization method

In order to describe the stress state of the part, the whole nomenclature of aircraft engine main operation modes corresponding to the aircraft flight envelope is considered. There are 26 loads patterns identified for each of the 8 brackets mounted on the engine. The maximum loadings in each possible direction were considered to facilitate the task. The bracket fixing in bolt joints was prescribed as boundary conditions, with a possibility for tangential sliding being considered.

The simplification procedure in the initial topology of the part to be designed was performed to determine the definition domain of the part. The computational domain was extended within the prescribed dimensions which don't prevent the part from being installed into the joint connection.

The initial value of the optimized bracket weight chosen was $75 \%$ of the weight of the model with the initial geometry. This parameter can vary during optimization via changing of the critical volume fraction which ensures satisfaction of the stresses values limitation. Hereby, the main objective is to optimize the topology of the bracket of interest under working loading conditions and to reduce the weight of the structure by $25 \%$.

The bracket topology was optimized in the specialized software allowing the particularities of the investigated problem formulation to be considered. It resulted in a 3D non-parametrized zone of the optimal material distribution in the definition domain (Figure 2).

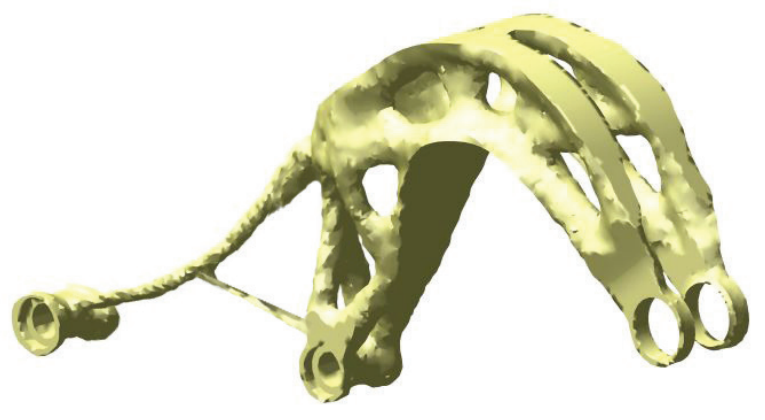

Fig. 2. The material distribution zone for the optimal bracket geometry

According to these results, the CAD model of the bracket with the optimal geometry was designed for the prescribed task conditions (Figure 3). The weight of the optimized bracket was 294 grams. Thus, the bracket weight was reduced by $25 \%$, which is 98 grams. Taking into account the number of this type brackets in the engine, the total gain in weight using the optimized bracket is 784 grams.

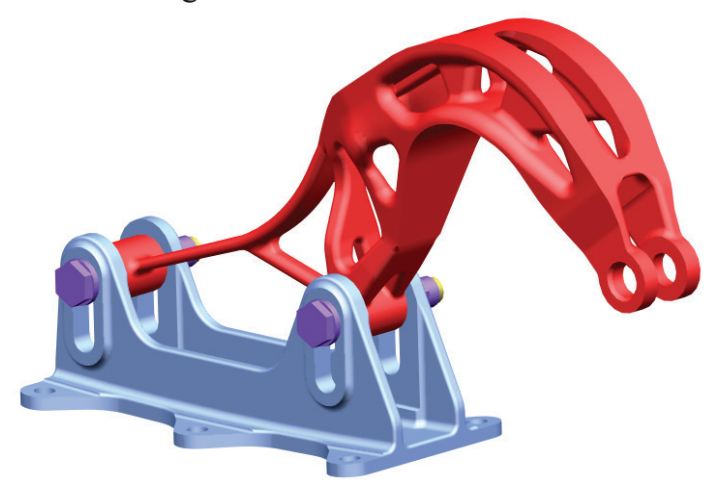

Fig. 3. The optimized CAD model of the hinge bracket The topological optimization method was verified in the virtual space via software package Ansys Workbench. 
The functional state of the bracket under investigation was simulated with the assistance of mathematical modelling at all the considered operational modes.

The maximum von Mises stress both in engineering, and in optimized model of the bracket was achieved under the same stress state pattern. Figure 4 shows the distribution of equivalent loadings under this load pattern in engineering and optimized bracket respectively.
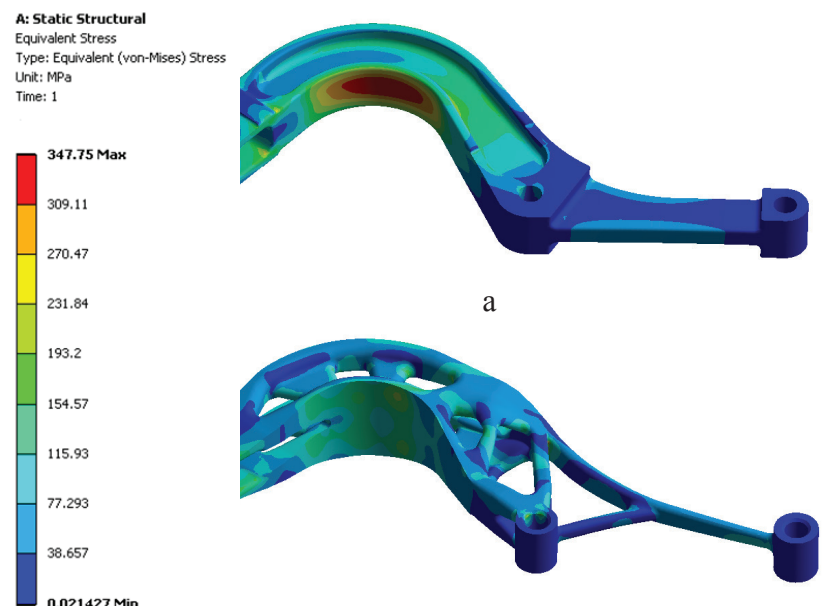

a

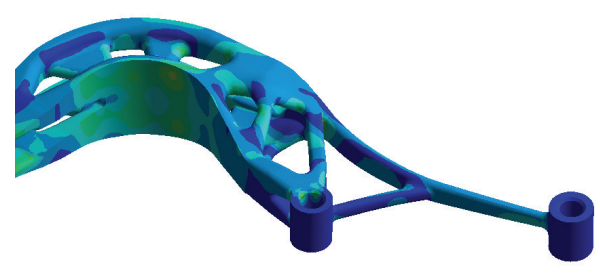

6

Fig. 4. The equivalent von Mises stresses in the maximum load pattern a) in the initial model, б) in the optimized model of the bracket

It may be noted that despite the $25 \%$ weight reduction, the optimized bracket is not only as good as the engineering model, but surpasses it in strength properties. The results of the optimization and verification calculations are given in Table 1.

Table 1. The Results of the Topological Optimization

\begin{tabular}{|c|c|c|c|c|}
\hline Model & $\boldsymbol{m}, \mathbf{k g}$ & $\boldsymbol{\sigma}, \mathbf{M P a}$ & $\boldsymbol{P ,} \%$ & $\boldsymbol{\delta}, \mathbf{\%}$ \\
\hline Initial model & 0.392 & 347 & 100 & - \\
\hline Optimized model & 0.294 & 228 & 74.9 & 34.35 \\
\hline
\end{tabular}

In Table $1, \mathrm{~m}$ is a weight of the bracket, $\sigma$ is the maximum von Mises stress, $P$ is a fraction of the initial bracket weight, $\delta$ is the level of the stress reduction in the bracket.

\section{Conclusions}

The topological optimization is an up-to-date effective method to create geometrical models and has a great application perspective in aircraft parts designing.

An optimized part is shape-intricate; such an approach to designing is often called a bionic design. It is technologically impossible to manufacture the similar geometry by conventional production methods. However, additive manufacturing methods allow for production of this bracket in one process step.

Currently, both bracket structures are ready for manufacturing by selective laser melting. The tooling compliant to the bracket operation loads is being developed for mechanical testing of the bracket.
This work was performed with financial support from Ministry of Education and Science of the Russian Federation (contract No. 02.G25.31.0168 dated 01.12.2015) within implementation of the RF Government Order No. 218 «On the measures of the State support for development of higher education institutions and organizations implementing integrated projects on hightech manufacturing build-up».

\section{References}

1. A.A. Inozemtsev, A.S. Dubrovskaya, K.A. Dongauser, N.A. Trufanov, Numerical Simulation of Cast Distortion in Gas Turbine Engine Components, IOP conference series: materials science and engineering, №84, 012039 (2015) DOI: 10.1088/1757-899X/84/1/012039

2. R.N. Faskhutdinov, A.S. Dubrovskaya, K.A. Dongauzer, P.V. Maksimov and N.A. Trufanov, Topology optimization of a gas-turbine engine part, IOP conference series: materials science and engineering, №177, 012077 (2017) DOI: 10.1088/1757-899X/177/1/012077

3. D.J. Brackett, I.A. Ashcroft and R: Hague, Topology optimization for additive manufacturing, 22nd Annual International Solid Freeform Fabrication Symposium, Texas, USA, 348-362 (2011)

4. A.A. Krotkikh, P.V. Maksimovl, The analysis of methods of refinement of the finite element model after topology optimization, International research journal, No 01 Vol. 55, pp. 91-94 (2017) DOI: 10.23670/IRJ.2017.55.071

5. E. Andreassen, A. Clausen, M. Schevenels, B. Lazarov, O. Sigmund, Efficient topology optimization in MATLAB using 88 lines of code, Structural and Multidisciplinary Optimization, 2010.:, DOI 10.1007/s00158-010-0594-7 